\title{
Application of Krill Herd and Water Cycle Algorithms on Dynamic Economic Load Dispatch Problem
}

\author{
Mani Ashouri \\ Babol Noshirvani University of Technology, Babol, 47148-71167, Iran \\ Email: mani_ashouri@yahoo.com \\ Seyed Mehdi Hosseini \\ Babol Noshirvani University of Technology, Babol, 47148-71167, Iran \\ Email: mehdi.hosseini@nit.ac.ir
}

\begin{abstract}
Dynamic economic dispatch (DED) is a complicated nonlinear constrained optimization problem and one of the most important problems in operation of power systems. In this paper two novel optimization algorithms have been proposed to be applied on DED problem. The first method, Krill herd (KHA) is a novel meta heuristic algorithm for solving optimization problems which is based on the simulation of the herding of the krill swarms as a biological and environmental inspired method and is applied on DED problem with two configurations named KHA1 and KHA2. The second algorithm is based on how the streams and rivers flow downhill toward the sea and change back in nature, named Water Cycle (WCA) method. Two common case studies considering various constraints have been used to show the effectiveness of these methods. The results and convergence characteristics show that the proposed methods are capable of giving high quality results which are better than many other previously applied algorithms.
\end{abstract}

Index Terms - Dynamic Economic Dispatch; Watercycle; Krill Herd; Optimization

\section{INTRODUCTION}

Your goal is to simulate the usual appearance of papers Dynamic economic dispatch (DED) is one of the most principal and serious issues in the operation of power systems. In DED, it's essential to obtain the most economical power dispatch of online generators to meet the predicted load demand over different periods of time while satisfying all operational and physical constraints. Actually DED is the extension of conventional economic dispatch (ED) problem, which schedules the output of the generators in sequential periods of time like 24 one hour periods, giving a whole day schedule. Traditional methods, commonly consider the fuel cost functions of generators as convex quadratic ones, which is not acceptable in reality.

Over the years, several attempts and studies have been proposed on solving DED problem considering various constraints and options which made DED problem more complicated and closer to the real situations. In the most general case these methods are classified in two categories of classical and meta-heuristic methods. Linear, non-linear, quadratic and dynamic programming are good examples of the first category[1]. However, these classical methods have convergence difficulties, specially on more complicated problems which cause the algorithm get stuck at local minima. Recently, stochastic methods such as Simulated annealing (SA)[2], Genetic algorithm (GA) [3], Particle swarm optimization (PSO) [3], Adaptive PSO (APSO) [4], Artificial bees colony (ABC)[3], MLS[5], IPS[6], BCO-SQP[7], ECE[8], Artificial immune system (AIS) [9], ,AHDE[1], Differential evolution (DE) [10], CDE3[11], have been proposed to solve different problems of DED. Although these heuristic methods usually provide reasonable solutions which can be achieved fast, but do not always guarantee discovering the globally optimal solution, giving results near global optimum, with long execution time when meeting more complicated problems with more local optima. So they cannot always lead to global optimum results because of their deficiency in calculation performance, solution quality or handling problems with more difficult formulation.

Recently new heuristic methods have been proposed which give better quality solutions in benchmark tests compared to many previous algorithms. Krill herd (KHA)[12] is a novel algorithm which is based on the simulation of the herding of the krill swarms as a biological and environmental inspired method. In KHA, krill individual positions are updated by three main components: movement led by other individuals, foraging motion and random physical diffusion, which are used along a Lagrangian model to update the movement of krill individuals. This method has fewer control variables, making KHA easy to implement. Water Cycle algorithm (WCA)[13] is another novel methods which is based on the natural behavior have water, cycling in nature.

The remainder of this paper is organized as follows: the formulation of DED problem is briefly described in section II, also explaining various DED constraints. In Section III the proposed KHA and WCA methods are described in detail. Two case studies have been considered to show the validity of the proposed methods in section IV, also proposing convergence characteristics, 
execution times and other details. Section $\mathrm{V}$ gives a brief discussion and finally the conclusions are extracted in section VI.

Any memberships in professional societies like the IEEE. Finally, list any awards and work for professional committees and publications. Personal hobbies should not be included in the biography.

\section{DED PROBLEM FORMULATION}

The goal of economic dispatch problem is to minimize the overall cost rate while meeting the load demand in different periods of time and satisfying various equality and inequality constraints which can be briefly described as follows:

\section{A. DED objective function:}

DED can be formulated as an optimization problem with the goal of minimizing the total power system generation cost, for $\mathrm{T}$ intervals as follows:

$$
\min \sum_{t=1}^{T} \sum_{i=1}^{N} F_{i}\left(P_{i}\right)
$$

where $N$ is number of generator units, $T$ is number of hours in research period, $P_{i}$ is the power output of the $i t h$ unit at time $t$ and $F_{i}$ is the production cost of the ith unit at the specific hour $t$, given below while considering valve point effect:

$$
F_{i}\left(P_{i}\right)=a_{i} P_{i}^{2}+b_{i} P_{i}+c_{i}+\left|e_{i} \sin \left(f_{i}\left(P_{i}-P_{i}\right)\right)\right|
$$

\section{B. Constraints:}

DED objective function is to be minimized subject to the following constraints:

1) Real power operating limits: Each unit has generation range, described as:

$$
P_{i}^{\min }<P_{i t}<P_{i}^{\max } \quad i=1,2, \ldots, N, t=1,2, \ldots, T
$$

where, $P_{i}^{\min }$ and $P_{i}^{\max }$ are the minimum and maximum generation limits for the ith unit in MW.

2) Real power balance constraint:

$$
\sum_{i=1}^{N} P_{i t}=P_{D t}+P_{L t} \quad t=1,2, \ldots, T
$$

where $P_{D t}$ is the total load demand in MW at time $t$ and $P_{L t}$ is the total transmission network loss which can be expressed using B-Coefficient matrix as follows:

$$
P_{L t}=\sum_{i=1}^{N} \sum_{j=1}^{N} P_{i t} B_{i j} P_{j t}+\sum_{i=1}^{N} B_{i 0} P_{i t}+B_{00}
$$

where $B$ is the loss coefficient matrix, $B_{i 0}$ is the linear term constant and $B_{00}$ is the transmission system constant. 3) Ramp rate limit constraints:

For each unit, output is limited by time dependent ramp rates at each hour and the generation may increase or decrease with corresponding upper and downward ramp rate limits to avoid undue thermal stresses on the boiler and the combustion equipment, as mentioned below:

$$
\left\{\begin{array}{l}
P_{i t}-P_{i(t-1)} \leq U R_{i} \\
P_{i(t-1)}-P_{i t} \leq D R_{i}
\end{array} \quad i \in N, t \in T\right.
$$

where $U R_{i}$ and $D R_{i}$ are the ramp up and down limits of the ith generator, respectively (MW/h).

4) Generators' prohibited operating zones:

Prohibited zones divide the operating region into disjoint sub regions. The generation limits for units with prohibited zones are:

$$
P_{i t} \in\left\{\begin{array}{l}
P_{i}^{\text {min }}<P_{i t}<P_{i 1}^{l} \\
P_{i, m-1}^{u}<P_{i t}<P_{i m}^{l} \\
P_{i, M_{i}}^{u}<P_{i t}<P_{i}^{\text {max }}
\end{array} \quad m=2,3, \ldots, M_{i}\right.
$$

\section{THE PROPOSED METHODS}

In this paper the application of two novel methods on DED has been proposed. The first algorithm is a new meta-heuristic optimization method for solving optimization tasks, which is based on the simulation of the herding of the krill swarms in response to particular biological and environmental processes named Krill Herd algorithm (KHA) [12]. The second proposed algorithm is another natural based method which is based on how the streams and rivers flow downhill toward the sea and change back, called water cycle algorithm (WCA)[13]. A brief explanation of the aforementioned methods is given in the remainder of this section.

\section{A. Krill Herd Algorithm:}

Krill herd (KHA)[12] is a novel meta-heuristic algorithm for solving optimization problems. KHA is based on the simulation of the herding of the krill swarms as a biological and environmental inspired method. The krill herds are swarms with no specific and parallel orientation which exist from hours to days and in $10 \mathrm{~s}$ to $100 \mathrm{~s}$ meters of space. When predators attack krill, they remove individual krill which results in reducing the krill density while Increasing density and finding areas of high food concentration are used as goals which finally lead the krill to herd around the global minima. Like many other algorithms, KHA is started with generating random krill individuals from the search space and then evaluating them. In GA and PSO Algorithms, arrays called "Chromosome" and "Particle Position" form the individuals carrying values of problem variables. In KHA, each array is called "Krill Individual", which $N_{P o p}$ numbers of them form the Krill matrix for a $n V a r$ dimensional optimization problem, shown below:

$$
\text { Krill Matrice }=\left[\begin{array}{cccc}
x_{1}^{1} & x_{2}^{1} & \ldots & x_{n V a r}^{1} \\
x_{1}^{2} & x_{2}^{2} & \ldots & x_{n V a r}^{2} \\
\ldots & \ldots & \ldots & \ldots \\
x_{1}^{N_{P o p}} & x_{2}^{N_{P o p}} & \ldots & x_{n V a r}^{N_{P o p}}
\end{array}\right]
$$

where, $N_{\text {Pop }}$ is number of krill individuals and $n V a r$ defines number of variables. 
The position of an individual krill in 2D space changes depending on time (iteration), based on three main actions considered in this method: movement affected by other krill individuals, foraging activity and random diffusion. For a d-dimensional decision space, the following Lagrangian model has been adopted for KHA:

$$
\frac{d X_{i}}{d t}=N_{i}+F_{i}+D_{i}
$$

where, $N_{i}, F_{i}$ and $D_{i}$ are the motions led by other krill individuals, the foraging activity and the physical diffusion of the krill individual, respectively.

The krill individuals try to maintain a high density and move due to their mutual effects as it is obvious in nature. So the direction of motion induced, is defined and estimated from the local swarm density, a target swarm density, and a repulsive swarm density as given in equation below:

$$
N_{i}^{\text {new }}=N^{\max } \alpha_{i}+\omega_{n} N_{i}^{\text {old }}
$$

where $\alpha_{i}=\alpha_{i}^{\text {local }}+\alpha_{i}^{\text {target }}$ and $N^{\max }, \omega_{n}, N_{i}^{\text {old }}$, $\alpha_{i}^{\text {local }}$ and $\alpha_{i}^{\text {target }}$ are the maximum induced speed, the inertia weight of the motion induced in $[0,1]$, the last motion induced, the local effect provided by the neighbors, and the target direction effect provided by the best krill individual, respectively. The effect of the neighbors in a krill movement individual can be assumed as mutual forces between individuals, determined as follows:

$$
\alpha_{i}^{\text {local }}=\sum_{j=1}^{N N} \frac{K_{i}-K_{j}}{K^{\text {worst }}-K^{\text {best }}} \frac{x_{j}-x_{i}}{\left\|x_{j}-x_{i}\right\|+\varepsilon}
$$

where $K^{\text {best }}, K^{\text {worst }}, K_{i}, K_{j}, x, N N$, and $\varepsilon$ are the best and the worst fitness values of the krill individuals, the fitness value of the ith krill individual, the fitness value of neighbor individual, the related positions, number of neighbors and a small positive number for avoiding the singularities, respectively. A sensing distance should be determined around a krill individual to find and choose the closest individuals, which can be found using the following equation after each iteration:

$$
d_{s, i}=\frac{1}{5 N} \sum_{j=1}^{N}\left\|x_{i}-x_{j}\right\|
$$

where $d_{s, i}$ and $N$ are the sensing distance for the ith krill individual and the number of the krill individuals, respectively. If the distance of two krill individuals is less than $d_{s, i}$, they are assumed to become neighbors. The effect of the individual krill with the best fitness on the ith individual krill can be determined using the following equation:

$$
\alpha_{i}^{\text {target }}=C^{\text {best }} K_{i}^{\text {best }} X_{i}^{\text {best }}
$$

where $C^{\text {best }}$ is the effective coefficient of the krill individual with the best fitness to the ith krill individual, defined as:

$$
\mathrm{C}^{\text {best }}=2\left(\operatorname{rand}+\frac{\mathrm{I}}{\mathrm{I}_{\max }}\right)
$$

Where rand, $I$ and $I_{\max }$ are a random number in the range $[0,1]$, current iteration and total number of iterations, respectively.

The foraging motion is based on two parameters similar to many other swarm based methods: the food location and the previous experience about that. So the formulation of the foraging motion is given below:

$$
\begin{aligned}
& F_{i}=V_{f} \beta_{i}+\omega_{f} F_{i}^{\text {old }} \\
& \text { where } \beta_{i}=\beta_{i}^{\text {food }}+\beta_{i}^{\text {best }}
\end{aligned}
$$

The third action is considered to be a random process and can be expressed in terms of a maximum diffusion speed and a random directional vector:

$$
D_{i}=D^{\max } \delta
$$

Where $D^{\max }$ and $\delta$ are the maximum diffusion speed and the random directional vector with arrays based on random values between -1 and 1 . According to the above mentioned motions the position of each krill individual gets closer to the global fitness. The thing that make KHA algorithm powerful is the parallel work of two global and two local strategies for the first two motions. So the position vector of a krill individual after the interval $\Delta t$ equals to:

$$
X_{i}(t+\Delta t)=X_{i}(t)+\Delta t \frac{d X_{i}}{d t}
$$

$\Delta t$ works as a scale factor of the speed vector and completely depends on the search space, obtained from the following formula:

$$
\Delta t=C_{t} \sum_{j=1}^{N V}\left(U B_{j}-L B_{j}\right)
$$

In order to improve the performance of the algorithm, crossover and mutation reproduction operators are taken from classical DE and genetic algorithms, which are not mentioned in order to summarize the paper.

\section{B. Water cycle algorithm:}

This novel nature inspired algorithm introduced in [13], is based on how the streams and rivers flow downhill toward the sea and change back. Water moves downhill in the form of streams and rivers starting from high up in the mountains and ending up in the sea. Streams and rivers collect water from the rain and other streams on their way downhill. The rivers and lakes water is evaporated when plants give off water as transpire process. Then clouds are generated when the evaporated water is carried in the atmosphere. These clouds condense in the colder atmosphere and release the water back in the rain form, creating new streams and rivers. Again, this method begins with an initial randomly generated population too, called "raindrops" resulting from rain or precipitation, similar to the given matrix in. The best raindrop is chosen as sea, a number of good raindrops as rivers and the rest of them are considered as streams flowing to rivers or directly to the sea. 


$$
\begin{gathered}
N_{\text {sr }}=\text { Number of rivers }+\underbrace{1}_{\text {Sea }} \\
N_{\text {Streams }}=N_{\text {Pop }}-N_{\text {sr }}
\end{gathered}
$$

Streams are assigned to the rivers and sea depending on the intensity of the flow calculated with the equation below:

$$
N S_{n}=\operatorname{round}\left\{\frac{\text { Cost }_{n}}{\sum_{i=1}^{N S_{r}} \operatorname{Cost}_{i}} \times N_{\text {Streams }}\right\}
$$

Where $N S_{n}$ is the number of streams which flow to a specific river or the sea.

The movement of a stream's flow to a specific river is applied along the connecting line between them using a randomly chosen distance, meaning $X \epsilon(0, C \times d)$. Where $C$ gets a user defined value between 1 and 2 and $d$ is the current distance between stream and river. If the value of $C$ be greater than 1 , the streams gain ability to flow in different directions toward the rivers. So the best value for $C$ may be chosen as 2 . This concept can also be used in flowing rivers to the sea. So new position for streams and rivers can be calculated using:

$$
\begin{aligned}
X_{\text {Stream }}^{i+1}= & X_{\text {Stream }}^{i}+\text { rand } \times C \\
& \times\left(X_{\text {River }}^{i}-X_{\text {Stream }}^{i}\right) \\
X_{\text {River }}^{i+1}= & X_{\text {River }}^{i}+\text { rand } \times C \times\left(X_{\text {Sea }}^{i}-X_{\text {River }}^{i}\right)
\end{aligned}
$$

Where rand is a uniformly distributed random number between 0 and 1 . If any streams solution value is better than its connecting river, their position is changed (the stream becomes river and the corresponding river is considered as a stream). Also the position of sea and a river is changed if the river has a better solution than the sea.

The evaporation process has an important role in the algorithm preventing from getting trapped in local optima and rapid convergence. The concept of this process is taken from the evaporation of water from sea while plants transpire water during photosynthesis. Then clouds are formed from the evaporated water and release them back to the earth in the form of rain and make new streams and rivers flowing to the sea. When the distance between the river and sea is less than a small number named $d_{\text {max }}$ the river has joined the sea and the evaporation process is applied, then the raining process will happen. $d_{\max }$ controls the search depth, near the sea. When a large value of $d_{\max }$ is selected, the search intensity is being reduced but its small value encourages it. The value of $d_{\text {max }}$ decreases linearly at the end of each iteration.

The raining process is similar to the mutation operator in GA. The new randomly generated raindrops form new streams in different locations. Again the raindrop with the best function value among other new raindrops is considered as a river flowing to the sea. The rest of them are considered as new streams which flow to the river or go directly to the sea. For the streams that directly flow to the sea a specific equation which increases the exploration near sea is used, resulting improvements in the convergence rate and computational performance of the algorithm for constrained problems.

$$
X_{\text {Stream }}^{\text {new }}=X_{\text {Sea }}+\sqrt{U} \times \operatorname{randn}(1, n \text { Var })
$$

Where $\sqrt{U}$ represents the standard deviation and $U$ defines the concept of variance. In fact the value of $U$ shows the range of searching region near the sea and randn is a normally distributed random number. The most suitable value found for $U$ is 0.1 , while the higher values increases the possibility of quitting from feasible region and the lower values reduce the searching space and exploration near the sea.

\section{DED using the proposed methods}

The flowcharts of the proposed methods are merged together and depicted in Fig.1.

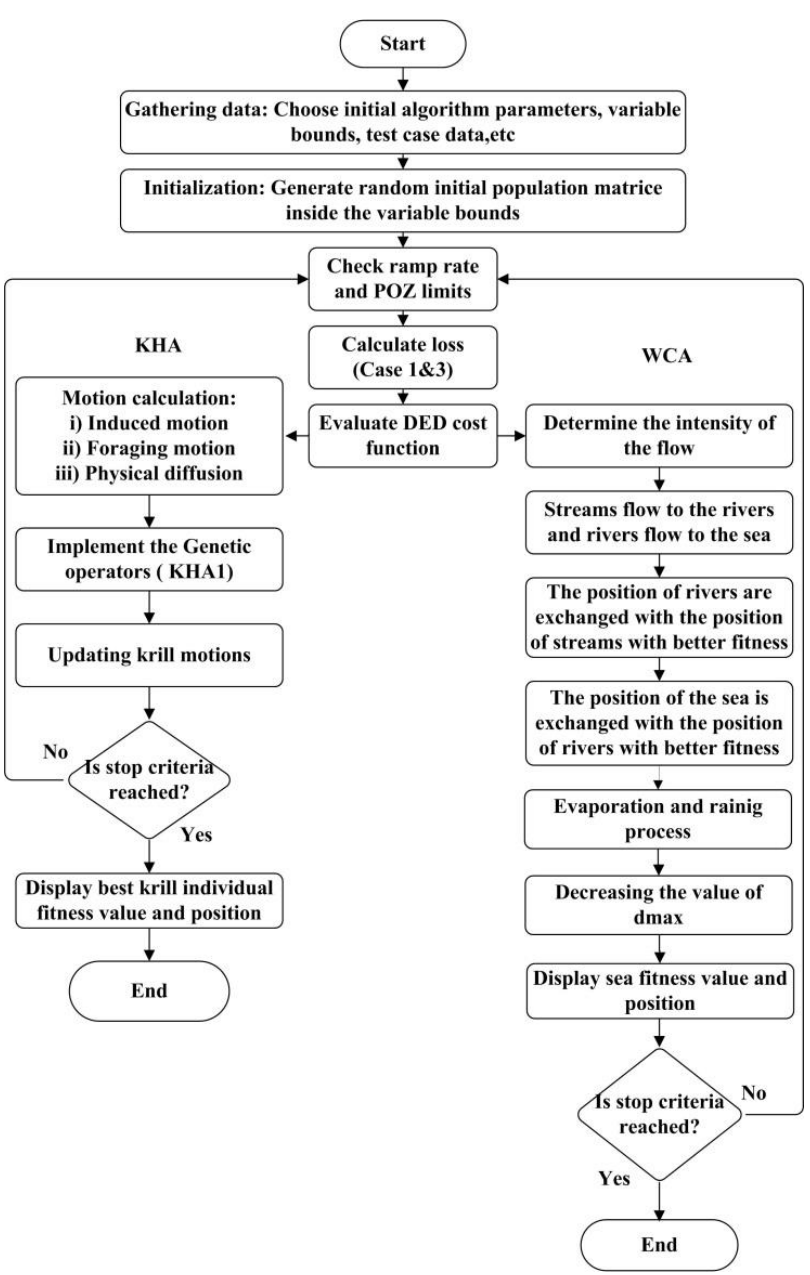

Fig 1. Flowchart of the proposed methods

\section{CASE STUDIES}

In this paper, two different case studies are considered to show the feasibility of the proposed methods. The first system is A 5 unit system with valve point loading effect considering transmission loss and prohibited zones. In the second case study a 10 unit system with valve point effect but neglecting transmission loss is considered as a bigger and more complicated test case. All tests and scripts are written in MATLAB software on a Pentium IV dual core pc with $2 \mathrm{~GB}$ of RAM. Results and convergence characteristics are given in the remaining of this section. 


\section{A. 5 Unit system}

The first case study consists of valve point loading effect, transmission loss and prohibited zones of generators with the data and $24 \mathrm{~h}$ load demand taken from[2]. The optimal algorithm parameters for KHA1 and KHA2 are $N_{\text {Pop }}=150, C_{t}=0.5, \omega_{n}=\omega_{f}=0.9$, $B_{i}^{\text {best }}=0.02, \alpha_{i}^{\text {best }}=0.01$ and for WCA are $N_{\text {Pop }}=$ $200, N S_{r}=40, d_{\max }=0.1, C=2, U=0.1$. Table 1 shows the 24 hour dispatch of the units for KHA1. Table 2 also gives the minimum, average and maximum costs of three proposed methods in comparison with some other previously applied methods on this case study. The convergence characteristics of three methods are depicted in Fig. 2 The distribution of objective function for 100 trials are given in Fig. 3 and Fig.4 for KHA1 and WCA methods, respectively.
As it is given in the table, KHA1 had the best result between 3 methods with minimum cost of 42664.7744 . WCA was close to KHA1 with the total cost of 42777.8503. In multiple runs, WCA had better mean cost with the average of 43118.8376 in 100 runs. KHA2 had good results but not good enough in comparison with two other methods.

\section{B. 10 unit system}

The second case study is bigger than the previous one while only consists of valve point effects and the transmission loss is neglected. The system data for this case study is adapted from[10]. The optimal parameters for KHA1 and 2 are $N_{\text {Pop }}=180, C_{t}=0.5, \omega_{n}=\omega_{f}=$ $0.9, B_{i}^{\text {best }}=0.02, \alpha_{i}^{\text {best }}=0.01$ and for WCA are $N_{\text {Pop }}=220, N S_{r}=50, d_{\max }=0.1, C=2, U=0.1$. Table 3 compares the results of the proposed methods with other methods in literature.

Table 1. 24h Dispatch of Units for Case 1 for KHA1

\begin{tabular}{|c|c|c|c|c|c|c|}
\hline Hour & P1(MW) & P2(MW) & P3(MW) & P4(MW) & P5(MW) & Loss(MW) \\
\hline 1 & 15.398 & 72.812 & 60 & 119.266 & 146.148 & 3.4946 \\
\hline 2 & 16.325 & 75.214 & 70 & 126.465 & 151.046 & 3.8777 \\
\hline 3 & 17.686 & 90 & 77.24 & 136.789 & 158.127 & 4.6390 \\
\hline 4 & 21.117 & 80 & 99.832 & 160 & 175 & 5.8504 \\
\hline 5 & 23.212 & 93.105 & 113.251 & 160 & 175 & 6.5942 \\
\hline 6 & 23.557 & 93.951 & 116.063 & 182.281 & 200 & 7.8198 \\
\hline 7 & 25 & 98.526 & 140 & 195.735 & 175 & 8.3686 \\
\hline 8 & 25 & 99.377 & 140 & 198.454 & 200.202 & 9.0903 \\
\hline 9 & 25 & 104.935 & 143.87 & 214.833 & 211.464 & 10.0853 \\
\hline 10 & 30 & 106.042 & 146.676 & 218.11 & 213.671 & 10.5444 \\
\hline 11 & 30 & 107.969 & 151.58 & 223.837 & 210.236 & 11.0228 \\
\hline 12 & 30 & 110.38 & 157.715 & 231.001 & 200.202 & 11.7122 \\
\hline 13 & 30 & 106.042 & 146.676 & 218.11 & 200 & 10.5477 \\
\hline 14 & 30 & 104.357 & 142.389 & 213.101 & 175 & 10.1798 \\
\hline 15 & 25 & 99.377 & 140 & 198.454 & 175 & 9.2613 \\
\hline 16 & 17.223 & 90 & 100 & 180 & 200.202 & 7.1994 \\
\hline 17 & 20.923 & 90 & 98.737 & 180 & 214.899 & 6.6853 \\
\hline 18 & 25 & 97.705 & 125 & 193.126 & 207.554 & 7.8796 \\
\hline 19 & 25 & 99.377 & 140 & 198.454 & 200 & 9.1284 \\
\hline 20 & 25 & 106.621 & 148.158 & 219.842 & 214.89 & 10.5554 \\
\hline 21 & 30 & 103.038 & 140 & 209.195 & 207.554 & 9.8881 \\
\hline 22 & 23.383 & 93.503 & 114.934 & 180.956 & 200 & 7.8535 \\
\hline 23 & 20.262 & 90 & 94.214 & 156.724 & 171.717 & 6.1684 \\
\hline 24 & 18.07 & 90 & 60 & 139.548 & 160.065 & 4.9872 \\
\hline
\end{tabular}

Table 2. Results Comparison for Case 1

\begin{tabular}{|c|l|l|l|l|}
\hline \multirow{2}{*}{ Method } & \multicolumn{3}{|c|}{ Production cost (\$) } & \multirow{2}{*}{ CPU time (min) } \\
\cline { 2 - 4 } & \multicolumn{1}{|c|}{ Min. value } & \multicolumn{1}{|c|}{ Mean value } & \multicolumn{1}{|c|}{ Max. value } & \\
\hline SA[2] & 47356.0000 & - & - & 5.86 \\
GA[3] & $44,862.42$ & $44,921.76$ & $45,893.95$ & 3.3242 \\
PSO[3] & $44,253.24$ & $45,657.06$ & $46,402.52$ & 3.5506 \\
APSO[4] & 44678 & - & - & - \\
ABC[3] & $44,045.83$ & $44,064.73$ & $44,218.64$ & 3.2901 \\
MLS[5] & 49216.81 & - & - & 0.024 \\
IPS[6] & 46530 & - & - & 4.53 \\
KHA1 & 42664.7744 & 43426.0415 & 45023.885 & 3.021 \\
KHA2 & 43071.6547 & 45345.0234 & 47839.7625 & 2.997 \\
WCA & 42777.8503 & 43118.8376 & 44204.7961 & 4.122 \\
\hline
\end{tabular}




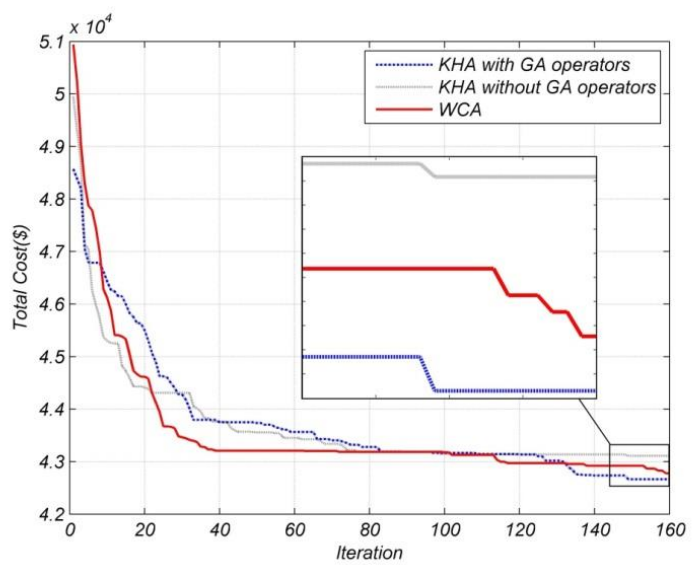

Fig. 2. Convergence characteristic curve for case-1.

Table 4 also shows the generation data for 24 hours resulted from KHA1. Figure 5, 6 and 7 demonstrate the convergence curve for this case study and the distribution of the objective function for 100 trial runs for KHA1 and WCA,respectively.

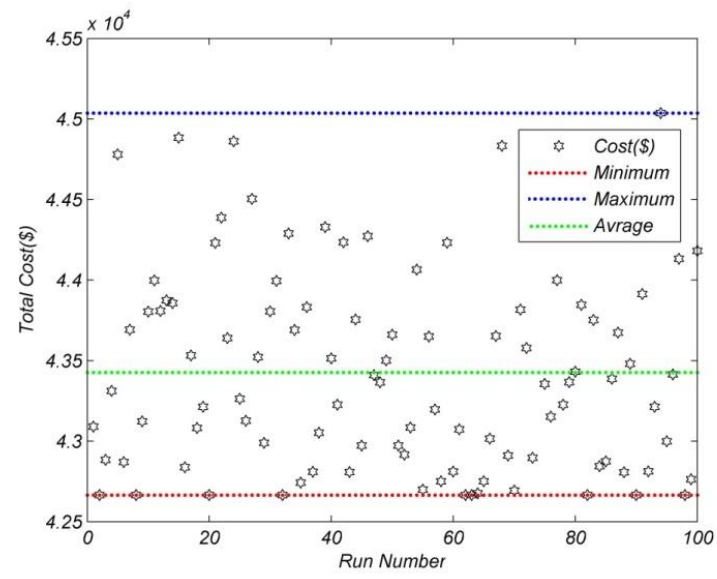

Fig. 3. Distribution of the objective function for 100 trial runs for 5-unit test system Using KHA1 (case 1)

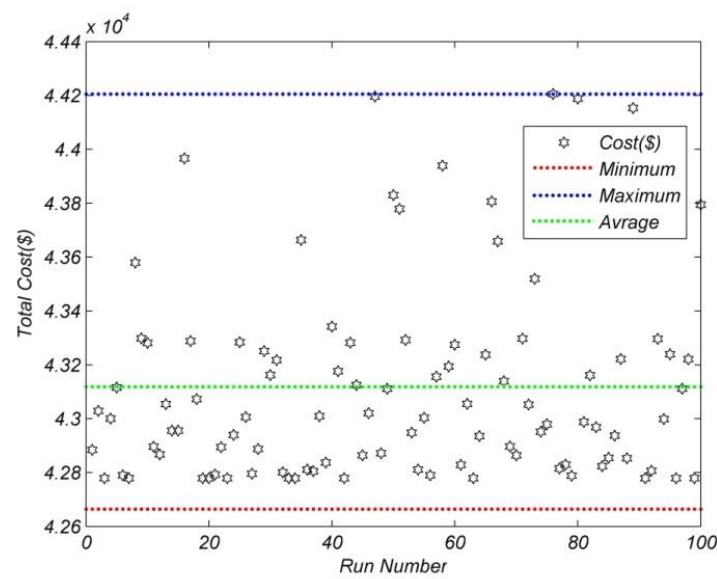

Fig. 4. Distribution of the objective function for 100 trial runs for 5 -unit test system Using WCA (case 1)

Again the best result has been achieved using KHA1 with the minimum cost of 1,018557.2407 and WCA with total cost of 1018622.2205 was the second best method. Both methods had much better results than many other previously applied ones. The average cost for 100 runs for WCA was better than KHA methods with the mean cost of 1019394.0194.

\section{DISCUSSION}

The execution time comparison between the methods shows that KHA had faster runs than WCA. That's because in WCA, the raining process happens when the streams reach to the river. The scripts written for this process in economic dispatch problem must calculate the euclidean distance between two $\mathrm{N}$ dimensional vectors, which $\mathrm{N}$ is the number of generation units.

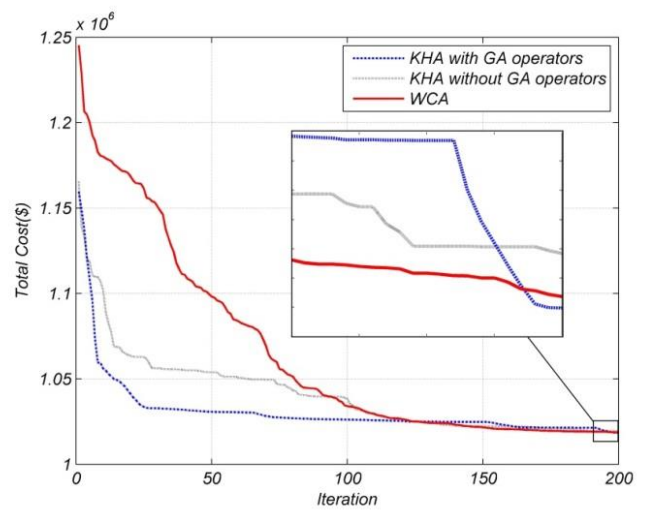

Fig. 5. Convergence characteristic curve for case-2

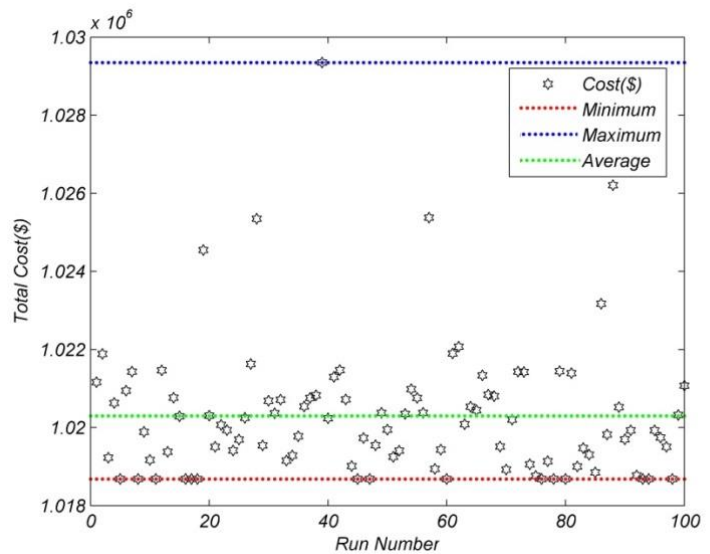

Fig. 6. Distribution of the objective function for 100 trial runs for 10 unit test system Using KHA1 (case 2)

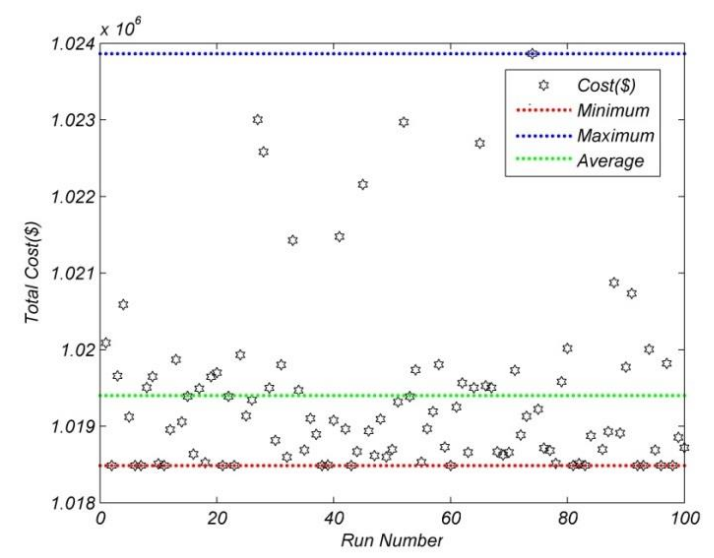

Fig. 7. Distribution of the objective function for 100 trial runs for 10 unit test system Using WCA (case 2) 
This process takes time on bigger units. As it can be seen form table 4 the difference between WCA and KHA,

CPU times is bigger compared to the 5unit system.

Table 3. Results Comparison for Case 2

\begin{tabular}{|l|l|l|l|l|}
\hline \multirow{2}{*}{ Method } & \multicolumn{3}{|c|}{ Production cost (\$) } & \multirow{2}{*}{ CPU time (min) } \\
\cline { 2 - 4 } & \multicolumn{1}{|c|}{ Minimum value } & \multicolumn{1}{|c|}{ Mean value } & \multicolumn{1}{|c|}{ Maximum value } & \\
\hline BCO-SQP[7] & $1,032,200.000$ & - & - & 3.24 \\
ECE[8] & $1,022,271.5793$ & $1,023,334.9297$ & - & 0.5271 \\
AIS[9] & $1,021,980.000$ & $1,023,156.000$ & $1,024,973.00$ & 19.01 \\
AHDE[1] & $1,020,082.000$ & $1,022,474.000$ & - & 1.10 \\
DE[10] & $1,019,786.000$ & - & - & 11.15 \\
CDE3[11] & $1,019,123.000$ & $1,020,870.000$ & $1,023,115.00$ & 0.32 \\
KHA1 & $1,018557.2407$ & 1020298.9975 & 1029345.2345 & 3.623 \\
WCA & 1018622.2205 & 1019394.0194 & 1023882.1018 & 7.3023 \\
KHA2 & 1019420.8208 & 1023847.3745 & 131453.8725 & 3.0112 \\
\hline
\end{tabular}

Table 4. 24h Dispatch of Units for Case 2 for KHA1

\begin{tabular}{|c|c|c|c|c|c|c|c|c|c|c|}
\hline Hour & P1(MW) & P2(MW) & P3(MW) & P4(MW) & P5(MW) & P6(MW) & P7(MW) & P8(MW) & P9(MW) & P10(MW) \\
\hline 1 & 150.0150 & 135.0000 & 192.1056 & 60.0000 & 122.9001 & 124.3728 & 129.6065 & 47.0000 & 20.0000 & 55.0000 \\
\hline 2 & 226.6120 & 135.0000 & 190.7530 & 60.0978 & 122.9175 & 122.9761 & 129.6282 & 47.0154 & 20.0000 & 55.0000 \\
\hline 3 & 303.1590 & 215.0000 & 182.9477 & 60.0000 & 122.8698 & 122.4487 & 129.5718 & 47.0000 & 20.0029 & 55.0000 \\
\hline 4 & 379.9372 & 222.2811 & 194.9529 & 60.9784 & 172.7400 & 123.4555 & 129.6208 & 47.034 & 20.0000 & 55.0000 \\
\hline 5 & 456.4919 & 222.2770 & 191.5980 & 60.6096 & 172.7047 & 124.7049 & 129.6051 & 47.0000 & 20.0089 & 55.0000 \\
\hline 6 & 456.5647 & 302.2770 & 209.4989 & 60.7132 & 222.6194 & 124.7292 & 129.5901 & 47.0000 & 20.0075 & 55.0000 \\
\hline 7 & 456.5002 & 309.4742 & 279.3874 & 60.0000 & 222.6079 & 122.4415 & 129.5888 & 47.0000 & 20.0000 & 55.0000 \\
\hline 8 & 456.4908 & 309.5506 & 301.8692 & 110.0000 & 222.6115 & 123.8669 & 129.6109 & 47.0000 & 20.0000 & 55.0000 \\
\hline 9 & 456.4751 & 389.5506 & 323.2665 & 120.4737 & 222.6368 & 160.0000 & 129.5919 & 47.0054 & 49.9941 & 55.0000 \\
\hline 10 & 456.4702 & 460.0000 & 320.8882 & 170.4737 & 222.5811 & 160.0000 & 129.5884 & 47.0044 & 52.0625 & 55.0000 \\
\hline 11 & 456.8587 & 460.0000 & 340.0000 & 220.4737 & 224.9827 & 160.0000 & 129.6224 & 47.0000 & 52.0625 & 55.0000 \\
\hline 12 & 456.4948 & 460.0000 & 339.6306 & 267.6389 & 222.5962 & 159.9882 & 129.6040 & 76.9988 & 52.0357 & 55.0000 \\
\hline 13 & 456.4955 & 396.8704 & 307.7255 & 241.3151 & 222.6984 & 124.9052 & 129.6372 & 85.3169 & 22.0357 & 55.0000 \\
\hline 14 & 456.5268 & 396.7835 & 292.2817 & 191.3151 & 172.7014 & 122.4642 & 129.5797 & 85.3120 & 20.0000 & 55.0000 \\
\hline 15 & 379.8493 & 396.7478 & 295.7526 & 168.4366 & 122.8689 & 122.4607 & 129.5895 & 85.2945 & 20.0000 & 55.0000 \\
\hline 16 & 303.2658 & 316.7478 & 302.0062 & 120.4340 & 73.0000 & 148.6514 & 129.5959 & 85.2990 & 20.0270 & 55.0000 \\
\hline 17 & 226.6673 & 396.7478 & 299.8748 & 70.4945 & 73.0508 & 123.2250 & 129.5896 & 85.3233 & 20.0000 & 55.0000 \\
\hline 18 & 303.2466 & 396.7913 & 297.3890 & 95.4079 & 122.8190 & 122.4268 & 129.5822 & 85.3373 & 20.0000 & 55.0000 \\
\hline 19 & 379.8830 & 396.7952 & 295.2565 & 119.0312 & 172.7362 & 122.4457 & 129.5792 & 85.2730 & 20.0000 & 55.0000 \\
\hline 20 & 456.4886 & 460.0000 & 313.9311 & 169.0234 & 222.6147 & 159.9978 & 129.6169 & 85.3274 & 20.0000 & 55.0000 \\
\hline 21 & 456.5388 & 396.7995 & 311.5649 & 121.2520 & 222.6192 & 125.2692 & 129.6277 & 85.3288 & 20.0000 & 55.0000 \\
\hline 22 & 379.7562 & 316.7995 & 275.2577 & 71.2520 & 172.7178 & 122.3637 & 129.5414 & 85.3118 & 20.0000 & 55.0000 \\
\hline 23 & 303.2398 & 236.7995 & 196.5695 & 60.0000 & 122.8791 & 122.5971 & 129.6063 & 85.3089 & 20.0000 & 55.0000 \\
\hline 24 & 226.6251 & 222.4868 & 186.1449 & 60.2484 & 73.0705 & 125.5110 & 129.6221 & 85.2913 & 20.0000 & 55.0000 \\
\hline
\end{tabular}

\section{CONCLUSION}

In this paper two novel heuristic algorithms have been applied on dynamic economic load dispatch problem. The first one, inspired from the movement of Antarctic krills, have been set as two sub-methods named KHA1 and KHA2 which differ in using and neglecting genetic operators. The second method named WCA, has been inspired from the cycling of water in the nature. The proposed methods have been applied on two common 5 and 10 unit DED case studies considering various DED constraints. Results, convergence characteristics and the distributions of DED objective function for 100 trial and runs for each methods show that WCA has more robustness giving great average results in multiple runs, while KHA1 gives unique best costs between multiple runs. Anyway both methods are totally new and powerful which can be applied on more complicated economic dispatch studies and also other optimization problems in power engineering studies.

\section{REFERENCES}

[1] Y. Lu, Q. Hui, L. Yinghai and Z. Yongchuan, "An adaptive hybrid differential evolution algorithm for dynamic economic dispatch with valve-point effect," Expert Syst. Appl., vol. 37, pp. 4842-9, 2010.

[2] CK. Panigrahi, R.N. Chakrabarti, M. Basu, "Simulated annealing technique for dynamic economic dispatch," Electr. Power Compon. Syst., vol. 34, pp. 577-86, 2006.

[3] S. Hemamalini, S.S. P, "Dynamic economic dispatch using artificial bee colony algorithm for units with valve-point effect," European Transactions on Electrical Power, vol. 21, pp. 70-81, 2011.

[4] B. Panigrahi, R. P, D. Sanjoy, "Adaptive particle swarm optimization approach for static and dynamic economic 
load dispatch," Energy Convers. Manage. vol. 49, pp. 1407-15, 2008.

[5] S. Hemamalini, S.S., "Dynamic economic dispatch using Maclaurin series based lagrangian method," Energy Convers. Manage. Vol. 51, pp. 2212-9, 2010.

[6] J Alsumait, Q. M., J Sykulski, A. Al-Othman, "An improved pattern search based algorithm to solve the dynamic economic dispatch problem with valve-point effect," Energy Convers. Manage., vol. 51, pp. 2062-7, 2010.

[7] M. B., "Hybridization of bee colony optimization and sequential quadratic programming for dynamic economic dispatch,” Electr. Power Energy Syst., vol. 44, pp. 591-6, 2013.

[8] A. I.S., "Enchanced cross-entropy method for dynamic economic dispatch with valve-point effects," Electr. Power Energy Syst., vol 33. pp. 783-90, 2011.

[9] S Hemamalini, S.S.P., "Dynamic economic dispatch using artificial immune system for units with valve-point effect," Elect. Power Energy Syst. vol. 33, pp. 868-74. 2011.

[10] R Balamurugan, S.S., "Differential evolution-based dynamic economic dispatch of generating units with valvepoint effects," Electr. Power Compo. Syst, vol. 36, pp. 828-43, 2008.

[11] Y. Lu, Z. J., Q. Hui, W. Ying and Z. Yongchuan, "Chaotic differential evolution methods for dynamic economic dispatch with valve-point effects," Eng Appl. Artif. Intel. vol. 24, pp. 378-87, 2011.

[12] A. Alavi, A.H.G, A. H., "KrillHerd: a new bio-inspired optimization algorithm," Communications in Nonlinear Science and Numerical Simulation, vol 17, pp. 4831-45, 2012.

[13] H. Eskandar, S.A., A. Bahreininejad, M. Hamdi, "Water cycle algorithm - A novel metaheuristic optimization method for solving constrained engineering optimization problems," Computers and Structure, vol 110-111, pp. 151-166, 2012.

\section{Authors' Profiles}

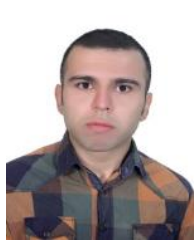

Mani Ashouri was born on September 20, 1987. He received his B.Sc. and M.Sc. degrees from Babol University of technology, Babol, Iran, in 2010 and 2012, respectively. In 2013, He joined TANESH Company as a protection and control engineer, testing and maintenance of transmission and substation relays, transformers and other protection and high voltage equipment. He is also extending his study career on all aspects of power system protection and control, DCS and SCADA systems, high voltage engineering, intelligent systems and optimization studies, generation dispatching, power flow problems and renewable energies.

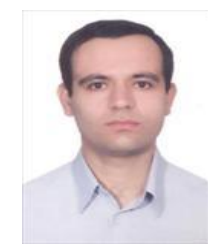

Seyyed Mehdi Hosseini was born in Sari, Iran, on September 18, 1978. He received the B.Sc. and M.Sc. degrees in Electrical Engineering in 2000 and 2002 and the Ph.D. degree in Electrical Engineering from Iran University of Science and Technology, Tehran, Iran in 2009. Presently, he is an Assistant Professor at Electrical Engineering Department of Babol Noshirvani University of Technology, Babol, Iran. His research interests are FACTS devices in transmission systems, application of distribution FACTS (D-FACTS) devices in distribution systems, distributed generation and reliability of distribution systems.

How to cite this paper: Mani Ashouri, Seyed Mehdi Hosseini,"Application of Krill Herd and Water Cycle Algorithms on Dynamic Economic Load Dispatch Problem", IJIEEB, vol.6, no.4, pp.12-19, 2014. DOI: 10.5815/ijieeb.2014.04.02 